\title{
Lugano Lymphoma Response Classification Partial Remission by PET
}

National Cancer Institute

\section{Source}

National Cancer Institute. Lugano Lymphoma Response Classification Partial Remission

by PET. NCI Thesaurus. Code C142796.

A score of 4 or 5 on a 5-point PET scale with reduced uptake compared with baseline and residual mass(es) of any size. Residual uptake higher than uptake in normal marrow but reduced compared to baseline. 